\title{
AS1387392, a novel immunosuppressive cyclic tetrapeptide compound with inhibitory activity against mammalian histone deacetylase
}

\author{
Satoshi Sasamura, Kazutoshi Sakamoto, Shoji Takagaki, Toshiko Yamada, Shigehiro Takase, Hiroaki Mori, \\ Takashi Fujii, Motohiro Hino and Michizane Hashimoto
}

The Journal of Antibiotics (2012) 65, 227; doi:10.1038/ja.2012.13

Addition to: The Jouranl of Antibiotics (2010) 63, 633-636; doi:10.1038/ ja.2010.51; published online 30 June 2010

It has come to the Editors' attention that background information relevant to these recently published articles was incomplete and confusing. Changes of the code name of the reportedly novel immunosuppressive cyclic tetra-peptide published in The Journal of Antibiotics, November issue, 63, 633-636 (2010), and the following three articles ${ }^{1-3}$ were not clearly explained. In addition, citations to the synthetic preparation and testing of those compounds were not included.

The code name 'AS1387392' used in these four papers represents the same compound as 'FR235225' (in papers published by the same authors in 2007 and $2008^{4,5}$ ) and 'WF27082E' (in the patent 'WO/ $2000 / 021979$ ' published in $2000^{6}$ ). This fermentation-derived compound is also identical to LGP1 that was prepared by total synthesis. ${ }^{7}$

The closely related natural product 'FR235222' was earlier reported in papers published in $2003^{8,9}$ and as 'WF27082B' in the above mentioned patent 'WO/2000/021979'.6 This code name of FR235222 has not changed.

In addition, the authors of the above four articles cited only the references that were limited to the natural products. However, the references that reported the synthetic endeavors ${ }^{7,10-12}$ to prepare analogs as better inhibitors of histone deacetylase also should have been included.

The journal apologizes to the readership for the confusion caused by the changes of the code names and the lack of citation of the earlier references during the review process.
1 Sasamura, S. et al. Bioconversion of AS1387392: screening and characterization of actinomycetes that convert AS1387392 to AS1429716. J. Antibiot. 63, 637-642 (2010).

2 Sasamura, S. et al. Bioconversion of AS1387392: bioconversion studies involving Amycolatopsis azurea JCM 3275. J. Antibiot. 63, 643-647 (2010).

3 Ueno, M. et al. Cloning and heterologous expression of P450Um-1, a novel bacterial P450 gene, for hydroxylation of immunosuppressive agent AS1387392. J. Antibiot. 63, 649-656 (2010).

4 Matsuoka, H. et al. Mechanisms of HDAC inhibitor-induced thrombocytopenia. Eur. J. Pharmacol. 571, 88-96 (2007).

5 Matsuoka, H. et al. Novel method for selecting immunosuppressive histone deacetylase (HDAC) inhibitors with minimal thrombocytopenia. Biol. Pharm. Bull. 31, 305-308 (2008).

6 Mori, H., Sakamoto, K., Tsurumi, Y., Takase, S. \& Hino, M. PCT Int. Appl. WO/2000/ 021979 A2 20000420 (2000).

7 D'Acunto, C. W. et al. LGP1, a histone deacetylase inhibitor analogue of FR235222, sensitizes promyelocytic leukemia U937 cells to TRAIL mediated apoptosis. Anticancer Res. 30, 887-894 (2010).

8 Mori, H. et al. FR235222, a fungal metabolite, is a novel immunosuppressant that inhibits mammalian histone deacetylase (HDAC). I. Taxonomy, fermentation, isolation, and biological activities. J. Antibiot. 56, 72-79 (2003).

9 Mori, H. et al. FR235222, a fungal metabolite, is a novel immunosuppressant that inhibits mammalian histone deacetylase (HDAC). II. Biological activities in animal models. J. Antibiot. 56, 80-86 (2003).

10 Terracciano, S. et al. Synthesis and biological activity of cyclotetrapeptide analogues of the natural HDAC inhibitor FR235222. Bioorg. Med. Chem. 18, 3252-3260 (2010).

11 Di Micco, S. et al. Molecular modeling studies toward the structural optimization of new cyclopeptide-based HDAC inhibitors modeled on the natural product FR235222. Bioorg. Med. Chem. 16, 8635-8642 (2008).

12 Gomez-Paloma, L. et al. Design and synthesis of cyclopeptide analogs of the potent histone deacetylase inhibitor FR235222. ChemMedChem 2, 1511-1519 (2007). 